\title{
ANÁLISIS SOCIAL DE UN GENOCIDIO: RUANDA 1994
}

\author{
ANÁLISE SOCIAL DE UM GENOCÍDIO: RUANDA 1994
}

\author{
Daniel Andrés Jiménez Montalvo ${ }^{1}$
}

\section{RESUMEN}

El presente artículo forma parte de la tesis, en desarrollo, "Genocidio en Ruanda: Un análisis multidimensional, el cual tiene como finalidad abordar un Análisis Social del Genocidio en Ruanda (1994). Dicho artículo se ha dividido en tres capítulos: el primer capítulo comprende los instrumentos analíticos. El segundo capítulo, desenvuelve el estudio de caso sobre el genocidio en Ruanda. Finalmente, se analiza y problematiza la actuación y las limitaciones de UNAMIR en el sostenimiento de la paz onusiana. En cuanto al referencial teórico se instrumentalizará la Teoría del Conflicto Social Prolongado de Edward Azar enfocando la discusión sobre las necesidades de los grupos comunales y el papel del Estado. En referencia a la metodología se hará uso del process tracing; se proseguirá mediante una revisión crítica de las fuentes secundarias sobre los principales acontecimientos en el genocidio de Ruanda; y se efectuará una revisión de las fuentes primarias online sobre la documentación de UNAMIR. Tornándose todo aquello evidenciado, mediante un análisis social que provee un cuadro de los síntomas agraviantes para la emergencia del genocidio y el fracaso de UNAMIR en Ruanda.

Palabras Clave: Ruanda, UNAMIR, Edward Azar, Paz, Genocidio.

\begin{abstract}
This work is part of the thesis, developing, "Genocide in Rwanda: A multidimensional analysis, which aims to address a Social Analysis of the Genocide in Rwanda (1994). This work has been divided into three chapters: the first chapter includes analytical instruments. The second chapter develops the case study on genocide in Rwanda. Finally, we analyze and problematize the performance and limitations of UNAMIR in onusiana sustaining peace. Regarding the theoretical framework of Social Conflict Theory shall be implemented Edward Azar Prolonged discussion focusing on the needs of community groups and the role of government. Referring to the methodology will make use of process tracing; will be pursued through a critical review of secondary sources on major developments in the genocide in Rwanda; and a review of the primary sources on-line documentation on UNAMIR be made. Turning everything evidenced by a social analysis that provides a picture of the aggravating symptoms for the emergence of genocide and the failure of UNAMIR in Rwanda.
\end{abstract}

Keywords: Rwanda, UNAMIR, Edward Azar, Peace, Genocide.

\footnotetext{
${ }^{1}$ Graduando en Relaciones Internacionales e Integración por la Universidad Federal de la Integración Latinoamericana. Correo institucional: daniel.montalvo@unila.edu.br / Correo personal: danielandres_360_@hotmail.com. Currículum lattes: http://lattes.cnpq.br/4893920506871914
} 


\section{INTRODUCCIÓN}

El presente trabajo forma parte de la tesis, en desarrollo, "Genocidio en Ruanda: Un análisis multidimensional, el cual tiene como finalidad abordar un Análisis Social del Genocidio en Ruanda (1994). Dicho trabajo se ha dividido en tres capítulos: el primer capítulo comprende los instrumentos analíticos provenientes de la Teoría del Conflicto Social Prolongado de Edward Azar, resaltando la importancia del factor social, y con especial énfasis en el contenido comunal como variable que juega un papel antagónico, contra el Estado, por su incapacidad de proveer las necesidades básicas de los grupos nacionales generando de tal forma la afluencia del conflicto violento. El segundo capítulo, desenvuelve el estudio de caso sobre el genocidio en Ruanda en dos partes: la primera efectuando una reconstrucción histórica desde 1970 hasta 1993 del territorio ruandés; y la segunda parte centrada en el desarrollo del genocidio de abril de 1994. Finalmente, se analiza y problematiza la actuación y las limitaciones de UNAMIR en el establecimiento del instrumento de peacekeeping para el sostenimiento y la preservación de la paz onusiana.

En cuanto al referencial teórico se instrumentalizará la Teoría del Conflicto Social Prolongado de Edward Azar en dos dimensiones: la primera dimensión abarca el génesis del conflicto destacando las variables de: contenido comunal; las necesidades humanas; gobernabilidad y el rol del estado; y las vinculaciones internacionales. Por su parte la segunda dimensión denominada proceso dinámico entrama tres variables: las acciones y estrategias comunales; las acciones y estrategias estatales; y los mecanismos incorporados del conflicto. En cuanto, a la metodología se hará uso haciendo uso del process tracing como instrumento fundamental de análisis cualitativo que permita la realización de un estudio sistemático para un diagnóstico a las pruebas recogidas y analizadas para conducir una investigación bajo preguntas e hipótesis planteadas por el investigador. De tal manera contribuyendo en la descripción de fenómenos políticos y sociales, y la evaluación de procesos causales.

Seguidamente, se efectuará a una revisión crítica de las fuentes secundarias en dos momentos: el primero, centrando la reconstrucción histórica de Ruanda desde 1962 hasta 1990, y en segunda instancia denotar los acontecimientos que 
desencadenaron el genocidio de 1990 hasta 1994. Posteriormente se realizará una revisión de fuentes primarias on-line sobre la documentación de UNAMIR proporcionada por el site de la ONU desde 1993 hasta 1996, centrándose en la revisión de las resoluciones efectuadas por el Consejo de Seguridad; los informes del Secretario General de la ONU; y los informe de la investigación independiente de las acciones de las Naciones Unidas durante el genocidio de 1994 en Ruanda. Tornándose todo aquello evidenciado, mediante un análisis social que provee un cuadro de los síntomas agraviantes para la emergencia del genocidio y el fracaso de UNAMIR en Ruanda.

\section{DIMENSIÓN TEÓRICA}

El presente trabajo reflexiona la importancia de del análisis social como variable que permea los síntomas que desencadenan los conflictos armados en África, siendo el objeto de estudio el análisis de caso del genocidio Ruandés en 1994 y la actuación de la misión UNAMIR de 1993 contra la dinámica del genocidio. Por cuanto, en un primer momento, el genocidio ruandés será examinado bajos los lentes analíticos de Edward Azar complementándose con las herramientas analíticas de Carlos Branco, Johan Galtung y Raphael Lemkin. Y en un segundo momento la misión UNAMIR, será norteada por los conceptos de peacekeeping y peacebuilding contendidos en la Doctrina Capstone.

El análisis del genocidio ruandés se permea por la Teoría de la Prolongación del Conflicto Social de Edward Azar de 1990. La cual se sustenta en 2 categorías: el génesis - siendo el primero -, el cual comprende un legado colonial presente en el contenido comunal $^{2}$ o sociedades multicomunales, formadas por medio del proceso colonial o la lucha intercomunal, caracterizados por la desarticulación entre el Estado y la sociedad (AZAR, 1990, p.7).

De tal forma, las sociedades multicomunales, adquieren una identidad por medio de un grupo, que identifica como necesidades esenciales la supervivencia física individual o comunal y el bienestar. Recordando que por un lado, la

\footnotetext{
${ }^{2}$ Azar, utiliza el término comunidad, como un término genérico para hacer referencia a grupos politizados quienes son miembros que comparten etnia, religión, lengua u otra identidad cultural característica.
} 
supresión de dichas necesidades generaría el nacimiento de un conflicto (ídem, p.8-9). Por tanto, el rol del Estado puede despojar o satisfacer las necesidades físicas, el acceso a las instituciones político-sociales o la aceptación de una identidad comunal. Pero optar por la extirpación de las necesidades básica, el Estado se transforma en la autoridad política monopolizada por un grupo dominante.

Siendo de este modo el esbozo de la lucha entre el monopolio de los grupos identitarios dominantes y los grupos comunales desprovistos de una entidad política justa y equitativa que promueva armonía y estabilidad ${ }^{3}$ (ídem, p. 10-11). Por tanto, al comprender grupos comunales desprovistos de sus necesidades básicas y el antagonismo de los grupos identitarios dominantes encarnados en el Estado, el conflicto social prolongado encuentra su génesis. Por lo cual, el Estado para contrarrestar el conflicto se delimitó a la vinculación internacional a través de la dependencia económica internacional y la política clientelar (ídem, p.11-12).

El proceso dinámico - segunda categoría -elucida factores responsables de la activación de conflictos sociales, siendo una de sus variables las acciones y estrategias comunales, que se nutren por la victimización individual que es reconocida colectivamente, a causa de quejas individuales consideradas en un cierto grado, de represivas o actos de supresión (ídem, p. 12-14). Las acciones y estrategias estatales que implementan la represión coercitiva o cooptación militar, con la finalidad de apaciguar las quejas comunales. Por lo cual, las acciones tanto comunales y estatales, se deterioran los canales de comunicación entre los contestantes hostiles y el Estado, permeando el conflicto social prolongado de miedo de la marginalización o menos integración comunal, siendo factores o mecanismos que se incorporan del conflicto (ídem, p. 15).

En una segunda instancia, Carlos Branco, demarca instrumentalización de las tensiones étnicas de los grupos comunales como justificativa de perpetuar el conflicto, identificando la identidad étnica como una construcción social, que surge de un proceso de socialización marcado por la presión de las élites de la

\footnotetext{
${ }^{3}$ De tal manera que Estados perpetuados por la prolongación de conflictos sociales, son incompetentes, regionalista, frágil y gobiernos autoritarios que fallan en la satisfacción de las necesidades básicas humanas.
} 
comunidad que instrumentalizan la etnicidad como una herramienta de competición social y política, es decir que la etnicidad posee un carácter contextual que puede ser moldeada en función de las condiciones estructurales de la sociedad (BRANCO, 2006, p. 134-135).

Por otro lado - en tercer lugar -, las tipologías de la violencia presentada por Johan Galtung, nortean tanto las acciones de los grupos comunales y las políticas de contención y de choque propuestas por el Estado. Siendo de esta manera la violencia galtungniana una variable latente expresa en el desencadenamiento del genocidio ruandés.

De tal manera, la violencia es considerada por Galtung (1969, p. 167), como el acto que "está presente cuando los seres humanos están siendo influenciados por lo que sus realizaciones somáticas y mentales reales están por debajo de sus posibles realizaciones". A dicho comprender la violencia en más amplias puede considerarse directa como una violencia manifiesta, siendo un aspecto visible de la violencia, que se muestra o expresa de una forma física, verbal o psicológica.

No sólo siendo evidente la violencia estructural muestra un tipo de violencia, más intrínseca que se presenta en los sistemas sociales, políticos y los económicos que hacen parte de una superestructura representada por la gobernanza de las sociedades, los Estados y el mundo (GALTUNG, 1998, p. 15-17). Y finalmente la violencia cultural conceptualizada como "aquellos aspectos de la cultura, en el ámbito simbólico de nuestra existencia, [...], que pueden utilizarse para justificar o legitimar la violencia directa o estructural" (GALTUNG, 2003).

Y finalmente - en cuarto lugar -, la concepción de genocidio de Raphael Lemkin (2009), reconfigura la visión del conflicto en Runda, trayendo a resaltar el tinte de constancia de las tensiones étnicas. Ante esto, Lemkin (2009, p.153), argumenta que genocidio entrama la destrucción de una nación o un grupo étnico. Proviene de la palabra griega genos (raza, tribu) y de la expresión latina cide (matar).

Señalando, una prosperidad de asesinatos masivos de la nación (todos los conformantes), pero este acto debe llevar un plan coordinado de diversas acciones con el objetivo de la destrucción de las bases esenciales de los diferentes grupos 
étnicos, entendidos como "miembros del grupo nacional", que serán atacados mediante "la destrucción del patrón nacional del grupo oprimido" y la "imposición del patrón nacional del opresor" (ídem, p.154).

El peacekeeping, es conceptualizado como la técnica designada para "preservar la paz" y sustentada por los peacemakers en cuanto a la ejecución de los acuerdos que nortearán la paz. De otra forma el peacekeeping ha desarrollado un modelo principalmente militar con el objetivo de observar los "altos al fuego, la separación de fuerzas después de las guerras interestatales y la paz sostenible" (UN, 2008, p.18).

En cuanto al peacebuilding, involucra la reducción del riesgo de vencer o sumir en el conflicto y el fortalecimiento de las "actitudes nacionales" para colocar la fundación de una "paz sostenible y desarrollo", dirigiendo sus efectos a las "causas enraizadas, estructurales del conflicto violento", transformándolo en uno más comprensivo y centrándose en el funcionamiento de la "capacidad del Estado de efectivizar y legitimar" sus funcionales para abrir los canales de comunicación entre el Estado y la sociedad. (Ídem, p.18)

\section{ENTRE EL GENOCIDIO Y LA PAZ: DE VÍCTIMAS A VERDUGOS}

El desencadenamiento de la revolución social - revolución ruandesa - de 1959 a 1961, consolidó la independencia de Ruanda en julio de 1962, trayendo consigo la inversión de la ideología ruandesa, que consagraba el poder de los hutus, plasmándose la "inversión del estigma socio-racial, tornándose el hutu [...] fundador de la organización social, al pasó que el tutsi por su lado, pasa a ser visto como un invasor extranjero" (BRUNETEAU, 2004, p. 250). Dicha revolución encarna la conjugación de los grupos comunales exponiendo la etnicidad que provoca la fragmentación de la sociedad.

De tal forma este poder se representó por Grégoire Kayibanda de etnia hutu bajo la política-programa de expulsión de los tutsis acusados por sus altas posiciones políticas (CASÓLIVA \& CARRERO, 2000) evidencia su violencia, en 1963 con la masacre de 14.000 tutsis y la expulsión de 250.000 (BRUNETEAU, 2004, p. 
251). Es decir que esta violencia directa, marca el poder un grupo étnico que juega un monopolio del Estado, y busca su poder en la masacre. No bastando el exilio de tutsis, en la primavera de 1972 en Burundi, provocó una insurrección hutu, desencadenando la represión militar de 100.000 víctimas hutus, dando como consecuencia el exilio de 200.000 hutus de Ruanda, generándose así campañas de pogromos $^{4}$ (ídem, p. 252). En tanto dichas campañas marcan por un lado una violencia estructural por las políticas de expulsión del territorio nacional, y una violencia directa representada por la muerte indiscriminada.

Así mismo en 1973 el gobierno del general Juvenal Habyarimana enfocado en la expulsión, como cuño de violencia estructural, de tutsis por programas de fuerzas hutus, hacia Uganda. Al crecer las "nuevas estructuras verticales" de etnicidad, de odio contra los tutsis, en 1987 que nace el Frente Patriótico Ruandés (FPR), con el objetivo de "imponer su derecho de regresar a Ruanda y de participar en la vida política ruandesa" ídem, p. 252). Ante ello, el primero de octubre de 1990, se desata un ataque del FPR contra el régimen de Habyarimana, a justificativa no su no permitido "retorno de los refugiados a causa de su pertenencia étnica" (CASÓLIVA \& CARRERO, 2000, p.7) y el "fin de la dictadura y de la ideología de exclusión "que genera refugiados" "(BRUNETEAU, 2004, p. 252). Desencadena la guerra civil, dura aproximadamente 3 años en los cuales en 1991 se perpetuaron masacres contra los bagowe, no bastando, el mero cesar fuego, se efectúan masacres contra los tutsis de Bugesera en 1992 (CASÓLIVA \& CARRERO, 2000, p.7). Cabe resaltar que las acciones y estrategias de los grupos nacionales resuenen en la naturalización de la violencia cultural, es decir las represiones de los grupos nacionales y las políticas de despojo de las instituciones políticoeconómicas que preserven la no marginalización de estos grupos de gran carga de etnicidad.

En la misma reproducción de violencia, en medida de contraposición al FPR, nace en 1992 las milicias Interhamwe 5 . Pero, sin dejar detrás la lucha el FPR despliega sus ataques hacia el centro y sur de Ruanda, siendo en 1993 diezmadas

\footnotetext{
${ }^{4}$ Es un ataque violento de carácter masivo contra las personas y sus bienes materiales. En una relevancia histórica este tipo de programas se utilizaron para masacrar a los judíos por la Alemania nazi hitleriana.

5 Es la emanación paramilitar del movimiento de juventud del partido presidencial" que inaugura los denominados escuadrones de la muerte: Red Zero y el Grupo Balas (BRUNETEAU, 2004, p. 255).
} 
las poblaciones de Ngarama, Mukingo, Kinigi, Kigombe, Matura, Kirambo (CASÓLIVA \& CARRERO, 2000, p.7). A tal punto que ante las masacres indiscriminadas bajo la presión internacional al presidente Habyarimana, en 1993 se firman los acuerdos de Arusha, en Tanzania para la consecución de un acuerdo político que establecía un gobierno de transición, la repatriación de refugiados, la integración de la fuerzas militares y nacionales, y el establecimiento de elecciones democráticas mediante el monitoriamiento UNAMIR en el despliegue de una operación de paz - de tipo peacekeeping -, en Ruanda en 1993 (ídem, p.7).

Siendo que UNAMIR encuentra su deterioro en sólo la configuración de un mero cesar fuego dando la apertura al resurgimiento del conflicto, por otro lado no constituye una misión centrada en la demarcación de entender las causas profundas del genocidio y no consolida una paz sostenible por la férrea demarcación de los grupos comunales con un alto grado de etnicidad.

El 6 de abril de 1994, tras las muertes de los líderes políticos hutus de Ruanda y Burundi, la Radio y Televisión Libre de Mille Collines (RTLM), difunde la idea de la muerte de los presidentes hutus, por el FPR ${ }^{6}$, desencadenando asesinatos por la milicia Interhamwe hutu y por el partido $\mathrm{MRND}^{7}$ y la guardia presidencial, hacia la ministra Agathe Uwilingiyimana y diez cascos azules de procedencia belga que tras el hecho se retiraron los contingentes belgas desprotegiendo el territorio ruandés (CASÓLIVA \& CARRERO, 2000, p.8), representando la inseguridad y la deteorización de la seguridad física del Estado (GARDNER, 2002, p.18) desencadenando una presencia total de anarquía (STEWART, 2008, p.12), que será contrarrestada, el 9 de abril, bajo el gobierno interino de Jean Kambanda (CASÓLIVA \& CARRERO, 2000, p.8), que consolidó la encarnación del terror hacia los tutsis, por medio de la instrumentalización de la etnia como medio represivo e inhibidor, expuesto en las diezmaciones de la población tutsi y la expulsión de todos los ciudadanos nacionales de Bélgica y Francia.

A cuestión de aquello los contingentes en territorio ruandés fueron retirados, dejando a la protección sin protección (ibídem). A este punto cabe mencionar que la victimización de los grupos comunales con lleva a la búsqueda de

\footnotetext{
${ }^{6}$ Frente Patriótico Ruandés.

${ }^{7}$ Movimiento Republicano Nacional por la Democracia y el Desarrollo. 
encarnar la falta del cumplimiento de sus necesidades básica por medio de violencia étnica, como válvula de escape al legado colonial.

A premeditación de las masacres, el 9 de abril se formó un gobierno interino encabezado en la imagen de Jean Kambanda (ibídem), que consolidó la encarnación del terror hacia los tutsis, de esa forma configura al Estado como un instrumento de un grupo étnico represivo e inhibidor. Por consecuencia de las diezmaciones del gobierno de Kambanda enfrentó la salida de todos los ciudadanos nacionales de Bélgica y Francia, y la intervención de la Cruz Roja Internacional en su ayuda humanitaria hacia las decenas de miles de ruandeses asesinados, todo aquello acontecido entre el 9 y 11 de abril del mismo año. La salida de los contingentes militares representó la inseguridad y la deteorización de la seguridad física del Estado y por otro lado generó vinculaciones internacionales humanitarias contra sus políticas represivas.

Al retirarse las tropas belgas de Ruanda, el 17 de abril, lejos de Kigali exactamente en Kibuye, donde comenzaron las filas de exterminio de la población tutsi. Dado los exterminios el 20 de abril el Secretario General de las Naciones Unidos, Butros Ghali, consciente de la ordena una acción inmediata y masiva de UNAMIR con la finalidad de la contención de las masacres por medio del reforzamiento de tropas adicionales y refuerzo del poder de la ONU. A este punto se reafirma las vinculaciones internacionales de tipo militar-político que provee de protección al Estado en detrimento de la autonomía e independencia del territorio ruandés.

Siendo así que en medio del terror, el mandato de UNAMIR se ajustó por el Consejo de Seguridad de la resolución 912, del 21 de abril de 1994, para su actuación como intermediaria en el conflicto de Ruanda con el objetivo de realizar un acuerdo para un alto al fuego, ayuda humanitaria, evolución institucional (política-económica y social), seguridad ciudadana. Complementándose con la denominada misión: Operación Turquesa para la salvación de civiles, soldados, oficiales y los milicianos de la región sudoccidental de Ruanda que participaron en el genocidio. A este punto, UNAMIR sólo centra su accionar en el cesar fuego del genocidio, y deja detrás su objetivo central la paz sostenible, como elemento esencial del peacekeeping. 
Las brigadas del FPR, formadas exclusivamente por jóvenes tutsis y desparramadas a lo largo de las colinas, fueron al principio el objetivo prioritario de las milicias hutus, pero progresivamente fueron los tutsis en general quienes fueron considerados como el enemigo a abatir (ibídem). A consecuencia del aumento de las masacres en el junio, el FPR, organizado en el nordeste del país, anuncia la salida de los extranjeros radicados en Ruanda, a consecuencia de un ataque por parte de la guerrilla tutsi: Ejército de Liberación de Ruanda (ALIR).

En su contraposición el Consejo de Seguridad autoriza temporalmente al gobierno francés la intervención para establecer el orden y crear un área de seguridad en la zona sur-oeste en Ruanda (ibídem). En falla de este punto, el accionar del peacekeeping se restringe a salvaguardar la seguridad de los grupos masacrados y deja de operacionalizar mecanismo que una paz sostenible en Ruanda.

Siendo a mediados del mes de julio, que el Frente Patriótico Ruandés se apodera de Kigali logrando el control militar de todo el territorio ruandés obligando al gobierno hutu radical a huir exiliarse a Zaire, cesando las matanzas el 4 de julio de 1994 y dando fin al conflicto (ibídem). Tras el alto el fuego y la instalación del nuevo Gobierno, las tareas de la UNAMIR se ajustaron aún más por el Consejo de Seguridad para garantizar la estabilidad y la seguridad en las regiones del noroeste y suroeste de Rwanda; alentar el retorno de la población desplazada; la proporción de la seguridad y apoyo a las operaciones de asistencia humanitaria dentro de Ruanda; y promover, a través de la mediación y los buenos oficios, la reconciliación nacional en Ruanda.

\section{UNAMIR: ¿ENFRENTANDO EL GENOCIDIO?}

Al no cesar las masacres el 20 de abril el Secretario General de las Naciones Unidos, Butros Ghali, ordena una acción inmediata y masiva de UNAMIR con la finalidad de contener las masacres por medio del reforzamiento de tropas, y la intervención de la ONU. Siendo así que en esta perspectiva se reafirman las vinculaciones internacionales de tipo militar-político (AZAR, 1990, p.15) que 
proveen seguridad y amparo a los tutsis, ante la fragmentación de la autonomía e independencia estatal encerrada en la violencia latente (GALTUNG, 1969, p.170).

Por cuanto, al persistir la violencia el mandato de UNAMIR es ajustado por el Consejo de Seguridad bajo la resolución 912, del 21 de abril de 1994, con la finalidad de participar arbitrariamente en el conflicto de Ruanda en preservación de los Acuerdos de Arusha para un alto al fuego, ayuda humanitaria, y la seguridad ciudadana (SC, 1994a). Siendo esta reajuste apoyado en la Operación Turquesa, como resguardo de las vidas de los civiles, soldados, oficiales y los milicianos de la región sudoccidental de Ruanda que vivenciaron el genocidio, pone en camino dinámico la reafirmación de la seguridad de los contingentes militares-civiles y expresando la importancia de la resolución del conflicto en el marco de los procesos de paz ratificados en los Acuerdos de Arusha (SC, 1994b).

Al dar un soporte de contención al conflicto UNAMIR, permite en el mes de mayo las movilizaciones de las brigadas del FPR, formadas exclusivamente por jóvenes tutsis y desparramadas a lo largo de las colinas, pero que fueron diezmadas por las milicias hutus (CASÓLIVA \& CARRERO, 2000, p.8), reactivando de esa forma la violencia, que en el mes de junio dará su respuesta a manos del FPR que desplegará una matanza de grupos hutus en colaboración con el Ejército de Liberación de Ruanda (ALIR). Por tanto, al no existir una solución al conflicto, el Consejo de Seguridad autoriza temporalmente al gobierno francés la intervención para establecer el orden y crear un área de seguridad en la zona sur-oeste en Ruanda. (ibídem). En falla de este punto, el accionar del peacekeeping se restringió a salvaguardar la seguridad de los grupos masacrados, dejando de lado su finalidad esencial: la paz sostenible en Ruanda (UN, 2008, p.18).

Por cuanto, tras aumentar la seguridad y la ayuda humanitaria en Ruanda, a mediados del mes de julio, el Frente Patriótico Ruandés se apodera de Kigali logrando el control militar de todo el territorio ruandés obligando al gobierno hutu radical a huir exiliarse a Zaire, cesando las matanzas el 4 de julio de 1994 y dando fin al conflicto (CASÓLIVA \& CARRERO, 2000, p.8). Permitiendo que tras el alto el fuego y la instalación del nuevo Gobierno, las tareas de la UNAMIR se ajustaran, por el Consejo de Seguridad, a las necesidades post-conflicto que garanticen la estabilidad y la seguridad en las regiones del noroeste y suroeste de Rwanda; la creación de políticas para el retorno de la población tutsis desplazada; la 
proporción de intensidad en asistencia humanitaria dentro de Ruanda; y la promoción de la mediación y los buenos oficios para la reconciliación nacional en Ruanda (UN, 2001).

\section{CONCLUSIONES}

En cuanto los instrumentos analíticos, la perspectiva de Edward Azar, el genocidio ruandés en su génesis esta enraizado a un legado colonial que se nutre por un patrón histórico de rivalidad y la lucha entre los actores comunales: tutsis y hutus. Esta desconformidad entre los grupos comunales genera la dominación de un grupo comunal, que en consonancia con el genocidio ruandés determina la realidad de la construcción de la nación dominada por los hutus dentro de la comunidad política, instrumentalizando al Estado como el actor perpetrador de la violencia cultural, como se presenció en los gobiernos Grégoire Kayibanda, Juvenal Habyarimana y Jean Kambanda. Pero, el monopolio de un solo grupo fragmenta la relación del Estado con los grupos comunales, e impide la construcción de instituciones que resguarden las necesidades de sobrevivencia e identidad de la población ruandesa.

Por otra parte, estos grupos comunales se forman por medio de su identidad que es socialmente construida, que comparte ampliamente lazos con la étnica, es decir que al crear lo hutus un imaginario de superioridad racial a consecuencia de su legado colonial y los tutsis crearan su ideario social por medio del Frente Patriótico Ruandés en memoria de su deteorización política-social y marginalización nacional, es que se consolida el repudio entre los grupos comunales dando cabida a que la trilogía de la violencia galtungniana permee el genocidio. De tal forma al fragmentarse los grupos comunales las necesidades humanas básicas como la supervivencia física comunal y el bienestar son violadas por la limpieza étnica perpetuada por los hutus en su trama de imposición de poder por medio de la coerción.

Y por último cabe resaltar la predominancia de las vinculaciones en la resolución y finalización del genocidio ruandés. En el ámbito nacional es Estado se manejó por la lógica de la superioridad de una grupo comunal, que naturalizó la 
violencia como medio de preservación de sus intereses y de sus integridad. Y en el ámbito internacional UNAMIR, al conjugar un peacekeeping, en la realidad ruandesa enfocó su accionar en un cesar el fuego y buscar la asistencia y protección de los civiles tutsis, dejando el foco central de su esencia: la paz sostenible que podría haberse buscado en el acompañamiento total de Ruanda desde su independencia, consolidando una comunidad política democrática que pueda atender las necesidades de los grupos comunales y en una segunda instancia UNAMIR no consolidó un proceso evolutivo de un peacekeeping para un peacebuilding.

\section{REFERENCIAS}

AZAR, Edward. The Management of Protracted Social Conflict: Theory and Cases. Aldershot: Darthmouth Publishers. 1990. p. 1-17.

BRANCO, Carlos. Etnicidad e Violência Étnica: As diferentes abordagens teóricas e sua utilidade na gestão dos conflictos. Relações Internaciones. 2006. p. 129-150.

BRUNETEAU, Bernard. o etnicismo genocidário do Pós-Guerra Fría e o advenimento de uma Jurisdição Internacional Permanente. In: _- 0 século dos genocidios da Arménia ao Ruanda. Tadinense AG, Lisboa. 2004. p. 245-259.

CASÓLIVA, Joan \& CARRERO, Joan. El África de los Grandes Lagos; Diez años de sufrimiento, destrucción y muerte. Published by Cristianisme i Justicia, Barcelona, 2000. p. 1-25.

GARDNER, Anne-Marie. Diagnosing Conflict: What do we know?. In: Hampson, F. O.; Malore, David (eds). From Reaction to Conflict Prevention: Opportunities for the UN System. Lynne Rienner Publishers. London. 2002. p.15-40.

GALTUNG, Johan. Tras la violencia, 3R: reconstrucción, reconciliación, resolución. Afrontando los efectos visibles e invisibles de la guerra y la violencia. Bilbao, Gernika Gogoratuz. 1998.

Twenty-Five Years of Peace Research: Ten Challenges and Some Responses. Journal of Peace Research. 22 (2), 1985. p. 141-185.

Violence, Peace, and Peach Research. Journal of Peace Research. 6 (3), 1969. p. 167-191.

Violencia cultural. Gernika Gogoratuz. Documento $n^{\circ}$ 14. 2003. p. 4-27. 
LEMKIN, Raphael. Genocidio. In: El dominio del Eje en la Europa ocupada: leyes de ocupación: análisis de la administración gubernamental: propuestas de reparaciones. Buenos Aires. Prometeo Libros. 2009. p. 153-173.

Informe Whitaker sobre la prevención y sanción del crimen del genocidio. In: __ El dominio del Eje en la Europa ocupada: leyes de ocupación: análisis de la administración gubernamental: propuestas de reparaciones. Buenos Aires. Prometeo Libros. 2009. p. 410-411.

SECURITY CONCIL. Resolution 912. 1994a. p.1-3. Disponible en: http://daccessdds-

ny.un.org/doc/UNDOC/GEN/N94/190/85/PDF/N9419085.pdf?OpenEleme nt. Acesso em: 25 de outbro de 2014.

Resolution 925. 1994b. p.1-4. Disponible en: http://daccess-ddsny.un.org/doc/UNDOC/GEN/N94/244/54/PDF/N9424454.pdf?OpenEleme nt. Acesso em: 25 de outbro de 2014.

STEWART, Frances. Horizontal inequalities and conflict: An introduction and some hypotheses. In: Horizontal inequalities \& conflict: Understanding group violence in multiethnic societies. Palgrave Macmillan. New York, 2008. p. 3-23.

UNITED NATIONS. Department of Peacekeeping Operations; Department of Field Support. Principles and guidelines [Doutrina Capstone]. New York, 18 jan. $2008 . \quad$ Disponivel em: http://www.peacekeepingbestpractices.unlb.org/Pbps/Library/Capstone_ Doctrine_ENG.pdf. Acesso em: 25 de outbro de 2014.

Rwanda-UNAMIR: Mandate. Peacekeeping missions. 2001. Disponivel em: http://www.un.org/en/peacekeeping/missions/past/unamirM.htm. Acesso em: 25 de outbro de 2014 . 